\title{
The expression of TLR2 and TLR4 in the kidneys and heart of mice infected with Acanthamoeba spp.
}

\author{
Karolina Kot ${ }^{1}$, Danuta Kosik-Bogacka ${ }^{2 *}$, Agnieszka Wojtkowiak-Giera ${ }^{3}$, Agnieszka Kolasa-Wołosiuk ${ }^{4}$ \\ and Natalia Łanocha-Arendarczyk ${ }^{1}$
}

\begin{abstract}
Background: Acanthamoeba spp. are cosmopolitan protozoans that cause infections in the brain, as well as extracerebral infections in the cornea, lungs and skin. Little is known about the mechanisms of the immunological response to these parasites in organs which are not their main biotope. Therefore, the purpose of this study was to determine the expression of TLR2 and TLR4 in the kidneys and heart of Acanthamoeba spp.-infected mice, with respect to the host's immunological status.
\end{abstract}

Methods: The mice were grouped into four groups: immunocompetent control mice; immunosuppressed control mice; immunocompetent Acanthamoeba spp.-infected mice; and immunosuppressed Acanthamoeba spp. infected mice. In the study, we used the amoebae T16 genotype which was isolated from a patient. The TLRs expressions in the kidneys and heart of mice were assessed by quantitative real-time polymerase chain reaction. Moreover, we visualized TLR2 and TLR4 proteins in the organs by immunohistochemical staining.

Results: In the kidneys, we observed a higher TLR2 expression in immunosuppressed mice at 24 days post-Acanthamoeba spp. infection (dpi) compared to the uninfected mice. There were no statistically significant differences in TLR4 expression in the kidneys between the immunocompetent and immunosuppressed mice, both of infected and uninfected mice. In the heart, we observed a difference in TLR2 expression in immunocompetent mice at $24 \mathrm{dpi}$ compared to immunocompetent mice at $8 \mathrm{dpi}$. The immunocompetent Acanthamoeba spp.-infected mice had higher TLR4 expression at 8 dpi compared to the immunocompetent uninfected mice.

Conclusions: Our results indicate that TLR2 is involved in response to Acanthamoeba spp. infection in the kidneys, whereas in the heart, both studied TLRs are involved.

Keywords: Acanthamoeba spp., Kidneys, Heart, Toll-like receptor 2 (TLR2), Toll-like receptor 4 (TLR4)

\section{Background}

Acanthamoebiasis caused by protozoans of the genus Acanthamoeba is an infection that is more frequently found in patients with a low immune response.

\footnotetext{
${ }^{*}$ Correspondence: kodan@pum.edu.pl

${ }^{2}$ Independent of Pharmaceutical Botany, Department of Biology and Medical Parasitology, Pomeranian Medical University in Szczecin, Powstancow Wielkopolskich 72, 70-111 Szczecin, Poland

Full list of author information is available at the end of the article
}

Development of this opportunistic infection is enhanced by chronic stress, coexisting diseases, and immunosuppressive drugs which are used to inhibit the rejection of transplanted organs [1]. It has been shown that corticosteroid therapy increases the number of parasites in the host [2]. Due to immunosuppression of the host organism, some parasitoses from asymptomatic or scarcely symptomatic infections leads to disseminated parasitic infections. These sudden multi-organ and multi-symptomatic changes may lead to the patient's death [3].

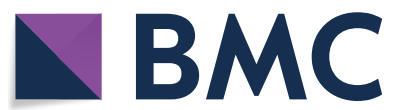

(c) The Author(s) 2020. This article is licensed under a Creative Commons Attribution 4.0 International License, which permits use, sharing, adaptation, distribution and reproduction in any medium or format, as long as you give appropriate credit to the original author(s) and the source, provide a link to the Creative Commons licence, and indicate if changes were made. The images or other third party material in this article are included in the article's Creative Commons licence, unless indicated otherwise in a credit line to the material. If material is not included in the article's Creative Commons licence and your intended use is not permitted by statutory regulation or exceeds the permitted use, you will need to obtain permission directly from the copyright holder. To view a copy of this licence, visit http://creativeco mmons.org/licenses/by/4.0/. The Creative Commons Public Domain Dedication waiver (http://creativecommons.org/publicdomain/ zero/1.0/) applies to the data made available in this article, unless otherwise stated in a credit line to the data. 
Acanthamoeba spp. trophozoites enter into the tissue or organs and induce granulomatous amoebic encephalitis (GAE), Acanthamoeba keratitis (AK) or disseminated acanthamoebiasis $[3,4]$. Disseminated acanthamoebiasis is predominantly confirmed post-mortem by histopathological examination of the organs and/or re-isolation of amoebae from tissue fragments because biochemical and hematological tests are difficult to interpret; blood parameters may be elevated, lower or not deviating from the norm $[5,6]$. In our earlier study concerning hematological and biochemical profiles in the blood of experimentally Acanthamoeba spp.-infected mice, we found only higher level of lymphocytes, monocytes, thrombocytes and aspartate aminotransferase $[7,8]$, despite the re-isolation of these amoebae from the brain, eyeball, lungs, kidneys, and spleen of the mice [7-11].

Mechanisms of renal and heart infection in acanthamoebiasis are still unknown. The immune response in renal and cardiac muscle cells to circulating antigens can be augmented by Toll-like receptors (TLRs), which play a key role in the non-specific immune response, recognizing pathogen-associated molecular patterns (PAMPs) present in pathogenic microorganisms [12]. The PAMPs on the membrane of Acanthamoeba spp. comprise proteins (33\%), phospholipids (25\%), sterols (13\%) and lipophosphoglycan (29\%) [13]. Moreover, TLR-signaling pathways might also be activated by components discharged by tissue damage or inflammation, or damageassociated molecular patterns (DAMPs), and heat-shock proteins (HSP) -60 and -70, which are revealed in Acanthamoeba spp. [14]. The recognition of these patterns by TLRs initiates the migration and aggregation of immunes cells at the site of infection, which in turn leads to the development of inflammation [15]. TLRs activate signaling pathways that lead to activation of the transcription factors NF- $\mathrm{kB}$ and IRFs, which control the expression of genes encoding proinflammatory cytokines, such as TNF- $\alpha$, IL- 8 and IFN- $\gamma[16,17]$. A lack of TLR2 and TLR4 on the immune cells leads to a delayed phagocytosis of pathogens, including bacteria such as Escherichia coli, Salmonella typhimurium and Staphylococcus aureus [18].

TLRs are involved in the response to several parasites, including Acanthamoeba spp. Alizadeh et al. [19] reported that Acanthamoeba spp. is recognized by TLR4, whereas other authors observed changes in TLR2 and TLR4 expression in the brain, lungs and eyes of Acanthamoeba spp.-infected mice [11, 20, 21]. However, no data exist on TLR activation in the organs and tissues which are not the main biotope of these protozoans. Therefore, the purpose of this study was to determine the TLR2 and TLR4 expression in the kidneys and heart of
Acanthamoeba spp.-infected mice, with respect to the host's immunological status.

\section{Methods \\ Isolation of Acanthamoeba spp.}

The Acanthamoeba spp. (AM22 strain) used in the study was isolated in 2009 from the bronchoaspirate fluid of a man with chronic leukaemia and atypical pneumonia. The patient had respiratory efficiency and acute septic shock. Moreover, interstitial changes with a visible pulmonary swelling were observed in the radiological examination. The AM22 strain was examined by molecular methods and the T16 genotype was detected [22]. Amoebae were kept on non-nutrient agar (NN agar) plates. Before the experiment, Acanthamoeba spp. were grown on NN agar plates with deactivated bacteria and incubated for $72 \mathrm{~h}$ at $37^{\circ} \mathrm{C}$ [9].

\section{Animal model}

The study was conducted on 96 male BALB/c mice about 6-10 weeks-old and an average weight of $23 \mathrm{~g}$. Mice were obtained from a licensed breeder (the Center of Experimental Medicine, Medical University in Białystok, Poland). During the experiment, the animals were in the Animal Facility of the Pomeranian Medical University in Szczecin, where they had constant access to fresh, clean water and Labofeed feed (Morawski, Kcynia, Poland), following recommended standards. Animals were housed in polycarbonate cages, and they were kept in standard laboratory conditions in a cycle of $12 \mathrm{~h}$ of light/12 $\mathrm{h}$ of darkness, at a temperature of $22 \pm 2{ }^{\circ} \mathrm{C}$, humidity of $56 \%$ and 15-20 air changes per hour.

The mice were divided into four groups: (i) immunocompetent uninfected mice: immunocompetent control group (C, $n=18$ ); (ii) immunocompetent Acanthamoeba spp.-infected mice (A, $n=30$ ); (iii) immunosuppressed by methylprednisolone sodium succinate uninfected mice: immunosuppressed control group (CS, $n=18$ ); and (iv) immunosuppressed by methylprednisolone sodium succinate Acanthamoeba spp.-infected mice (AS, $n=30$ ).

Animals belonging to groups AS and CS were given $0.22 \mathrm{mg}$ methylprednisolone sodium succinate (MPS; Solu-Medrol, Pfizer, Kent, UK) dissolved in $0.1 \mathrm{ml} 0.9 \%$ saline. MPS was administered intraperitoneally daily for 4 days prior to Acanthamoeba spp. infection. The drug was given to reduce immunity of the mice [11, 23]. Animals belonging to groups A and AS were administered intranasally $3 \mu \mathrm{l}$ of suspension consisting of 10,00020,000 trophozoite form of Acanthamoeba spp. Mice from the control groups ( $\mathrm{C}$ and $\mathrm{CS}$ ) were given the same volume of saline. The animals were sacrificed by pentobarbital sodium (Euthasol vet, FATRO, Raamsdonksveer, The Netherlands), administered at $2 \mathrm{ml} / \mathrm{kg} \mathrm{mg} / \mathrm{kg}$ body 
weight intraperitoneally at 8,16 , and 24 days post-Acanthamoeba spp. infection (dpi). The kidneys and heart were collected from the mice.

\section{TLRs expression}

TLR2 and TLR4 gene expression in the kidneys and heart of mice was assessed by quantitative real-time polymerase chain reaction using a Light Cycler real-time PCR detection system (Roche Diagnostic $\mathrm{GmbH}$, Mannheim, Germany) as recently described [11, 20]. Briefly, the increase in reaction product was assessed by increasing the fluorescence signal of SYBR Green I dye binding to double-stranded DNA. Target cDNA was quantified using a relative estimation method using a calibrator, which was prepared as a cDNA mix from all samples, and subsequent dilutions were used to create a standard curve according to the manufacturer's instructions (Roche Diagnostic GmbH). The housekeeping gene porphobilinogen deaminase was amplified as the reference gene for mRNA quantification. The amount of TLR2 and TLR4 transcripts in each sample was determined by the geometric mean of the transcript level of the housekeeping gene, and the mRNA levels of TLR2 and TLR4 are expressed as the multiplicity of cDNA concentrations in the calibrator.

\section{Immunohistochemical staining}

The paraffin-embedded sections $(3-5 \mu \mathrm{m})$ of kidneys and heart of control mice and hosts infected with Acanthamoeba spp. were stained to visualize TLR2 and TLR4 proteins. Immunohistochemistry was performed using specific primary rabbit polyclonal antibodies against TLR2 and TLR4 (Cat\# sc-10739 and sc-30002, respectively; Santa Cruz Biotechnology, Inc., Oregon, USA) at a 1:500 dilution. The procedure of immunohistochemical staining is described by Kot et al. [11]. Briefly, the sections were microwaved to recover antigenicity and then incubated with primary antibodies overnight at $4{ }^{\circ} \mathrm{C}$. Subsequently, the sections were stained with an avidin-biotin-peroxidase system with diaminobenzidine as the chromogen (Cat\# K0679; DakoCytomation Inc., Carpinteria, CA, USA). The sections were washed in distilled $\mathrm{H}_{2} \mathrm{O}$ and counterstained with hematoxylin. In negative controls, samples were not incubated with primary antibodies. Positive samples were determined microscopically by identifying brown pigmentation. Samples were evaluated using a light microscope (DM5000B; Leica, Wetzlar, Germany).

\section{Statistical analysis}

Statistical analysis was made using Microsoft Excel 2016 and StatSoft Statistica v10.0. Comparisons between intergroups were made using the MannWhitney U-test. The level of significance was $P<0.05$.

\section{Results}

\section{TLR2 and TLR4 expression in the kidneys}

The TLR2 expression in the kidneys of immunocompetent mice was similar to uninfected and Acanthamoeba spp.-infected mice. In immunosuppressed mice, we found a significantly higher level of TLR2 mRNA expression at 24 days post-Acanthamoeba spp. infection (dpi) compared to uninfected animals $(0 \mathrm{dpi} ; U=24, P<$ $0.05)$, and the levels obtained from mice at $8 \mathrm{dpi}(U=16$, $P<0.05$; Fig. 1). Additionally, there was a statistically significant difference in the TLR2 expression between the immunocompetent and immunosuppressed Acanthamoeba spp.-infected mice at $24 \mathrm{dpi}(U=20, P<0.05$; Fig. 1). The TLR4 expressions in the immunocompetent uninfected mice and mice at $8 \mathrm{dpi}, 16 \mathrm{dpi}$ and $24 \mathrm{dpi}$ were similar. The TLR4 mRNA expression in the immunosuppressed Acanthamoeba spp.-infected mice was higher at $16 \mathrm{dpi}$ and $24 \mathrm{dpi}$ compared to the immunosuppressed uninfected mice, but the differences were not statistically significant. Taking into account the immunological status of the hosts, we found statistically significant differences in the TLR4 expression between the immunocompetent and immunosuppressed mice at $16 \mathrm{dpi}$ and at $24 \mathrm{dpi}$ ( $U=12$ and $U=15, P<0.05$, respectively; Fig. 2 ).

The results of the immunohistochemical reaction showed changes in the intensity of TLR2 and TLR4 immunoexpression in the kidneys of Acanthamoeba spp-infected mice in comparison to the control groups (Fig. 3). In the immunocompetent and immunosuppressed uninfected mice, TLR2 expression was found in the proximal tubules (Fig. 3, white arrows), while after Acanthamoeba spp. infection brown pigmentation was observed in the distal tubules and collecting ducts (Fig. 3, black and red arrows, respectively). The TLR2 expression in the kidneys of the immunocompetent Acanthamoeba spp-infected mice remained at a similar level during the infection. In the immunosuppressed mice, the TLR2 expression intensity and the number of immunopositive cells were similar at $8 \mathrm{dpi}$ and $16 \mathrm{dpi}$ (Fig. 3). During the decline of infection, in the 24 dpi group TLR2 expression increased. The immunoexpression of TLR4 was observed in the proximal and distal tubules (Fig. 3, white and black arrows, respectively), collecting ducts (Fig. 3, red arrows) and in the renal corpuscles (Fig. 3, yellow arrows). The TLR4 expression in the immunocompetent Acanthamoeba spp--infected mice was similar to TLR2 expression and remained at a similar level during the infection. In 
the immunosuppressed Acanthamoeba spp.-infected mice, the number of TLR4 immunopositive cells and the intensity of TLR4 immunoreaction increased at $16 \mathrm{dpi}$ and then decreased at $24 \mathrm{dpi}$ (Fig. 3). The highest intensity of TLR4 immunoreaction was observed in the immunosuppressed mice at 16 days post-Acanthamoeba spp. infection.

\section{TLR2 and TLR4 expressions in the heart}

In the heart of the immunocompetent Acanthamoeba spp.-infected mice, the highest TLR2 expression was observed at $8 \mathrm{dpi}$. In the following days, TLR2 expression decreased. We found a statistically significant difference in the TLR2 expression level in the immunocompetent mice between 8 dpi and $24 \mathrm{dpi}(U=31.5, P<0.05$; Fig. 4). In immunosuppressed mice, no statistically significant differences were found in the TLR2 expression levels between infected and uninfected groups. The TLR4 expression was increased in immunocompetent mice at 8 dpi compared to uninfected animals and decreased in immunocompetent mice at $16 \mathrm{dpi}$ compared to mice at 8 dpi ( $U=23.5$ and $U=22.5, P<0.05$, respectively; Fig. 5). In the immunosuppressed mice, there were no statistically significant differences in the TLR4 expression levels between Acanthamoeba spp.-infected and uninfected mice. Comparing the TLR4 immunoexpression between immunocompetent and immunocompromised mice, we found the differences in uninfected animals and in the mice at 16 days post-Acanthamoeba spp. infection ( $U=23$ and $U=23, P<0.05$, respectively; Fig. 5).

Immunohistochemistry revealed changes in the TLR2 and TLR4 immunoexpression in the heart of immunocompetent Acanthamoeba spp.-infected mice compared to uninfected mice (Fig. 6). In the immunocompetent and immunosuppressed control mice, only some cardiomyocytes showed TLR2 expression (Fig. 6a and 6e, respectively). At the beginning of infection in immunocompetent mice, TLR2 expression was observed in most cardiomyocytes (Fig. 6b, c). The lowest immunohistochemical reaction in the cardiac muscles of immunocompetent mice was found at $24 \mathrm{dpi}$ (Fig. $6 \mathrm{~d}$ ). In the immunosuppressed Acanthamoeba spp.-infected mice, the highest intensity of TLR2 expression was noted at 8 and $16 \mathrm{dpi}$ as brown pigmentation in the most cardiomyocytes (Fig. 6f and 6g, respectively). In the 24 dpi group of immunosuppressed mice, TLR2 was expressed only in some cardiomyocytes (Fig. 6h). We did not find TLR2 immunopositive cardiomyocyte nuclei in the hearts of immunocompetent and immunosuppressed Acanthamoeba spp.-infected mice (Fig. 6). In the heart of immunocompetent Acanthamoeba spp.-infected mice, we observed increased TLR4 expression at $8 \mathrm{dpi}$, while at $16 \mathrm{dpi}$, we observed decreased number of TLR4-positive cells (Fig. 6). In the immunosuppressed Acanthamoeba spp.-infected mice, the highest TLR4 expression was observed in the hearts of mice at 8 and $16 \mathrm{dpi}$, while at $24 \mathrm{dpi}$ there was a decrease in TLR4 expression (Fig. 6p).

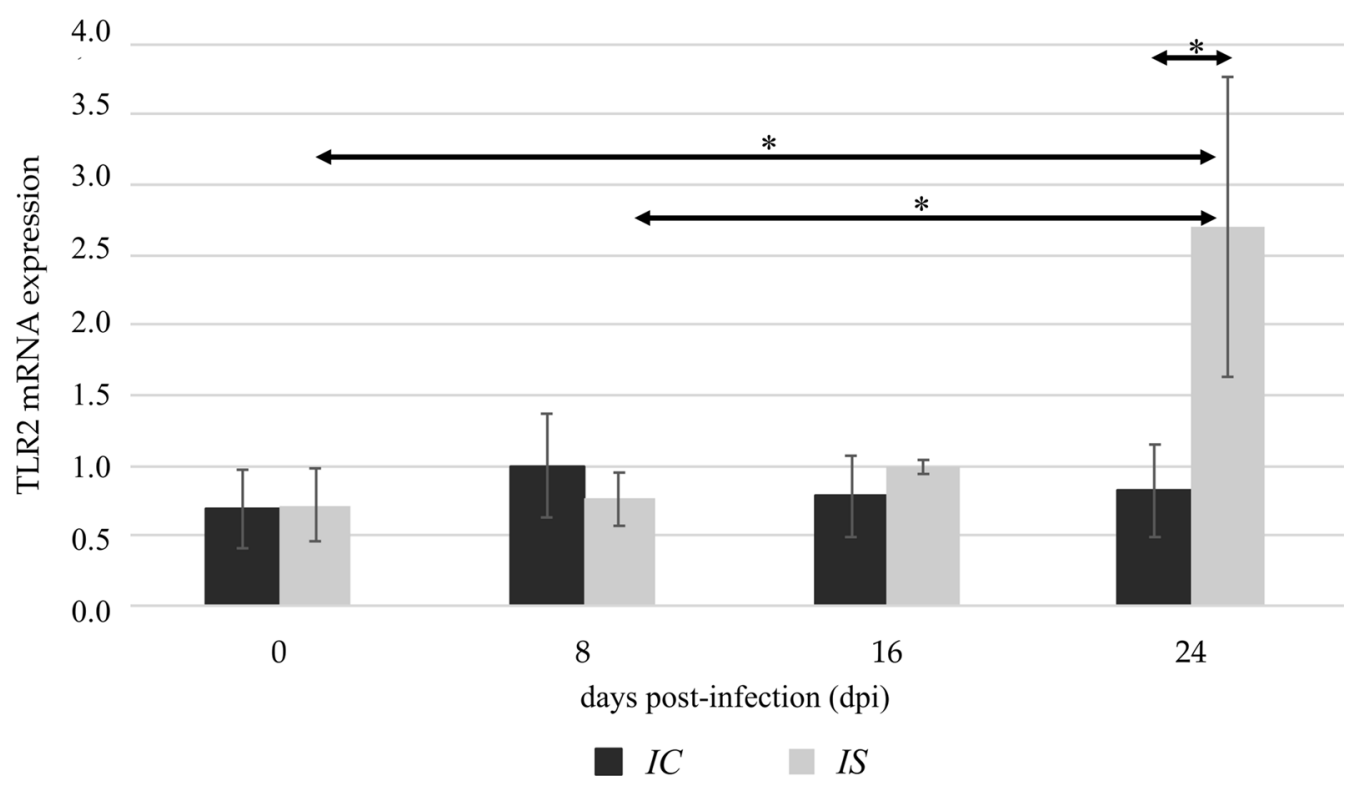

Fig. 1 The TLR2 mRNA expression in the kidneys of uninfected ( 0 dpi) and infected mice at 8, 16, and 24 dpi, in accordance with the immunological status of hosts (IC, immunocompetent mice; IS, immunosuppressed mice). The results are the means and standard deviations (SD) of six independent experiments; ${ }^{*} P<0.05$ using a Mann-Whitney U-test 


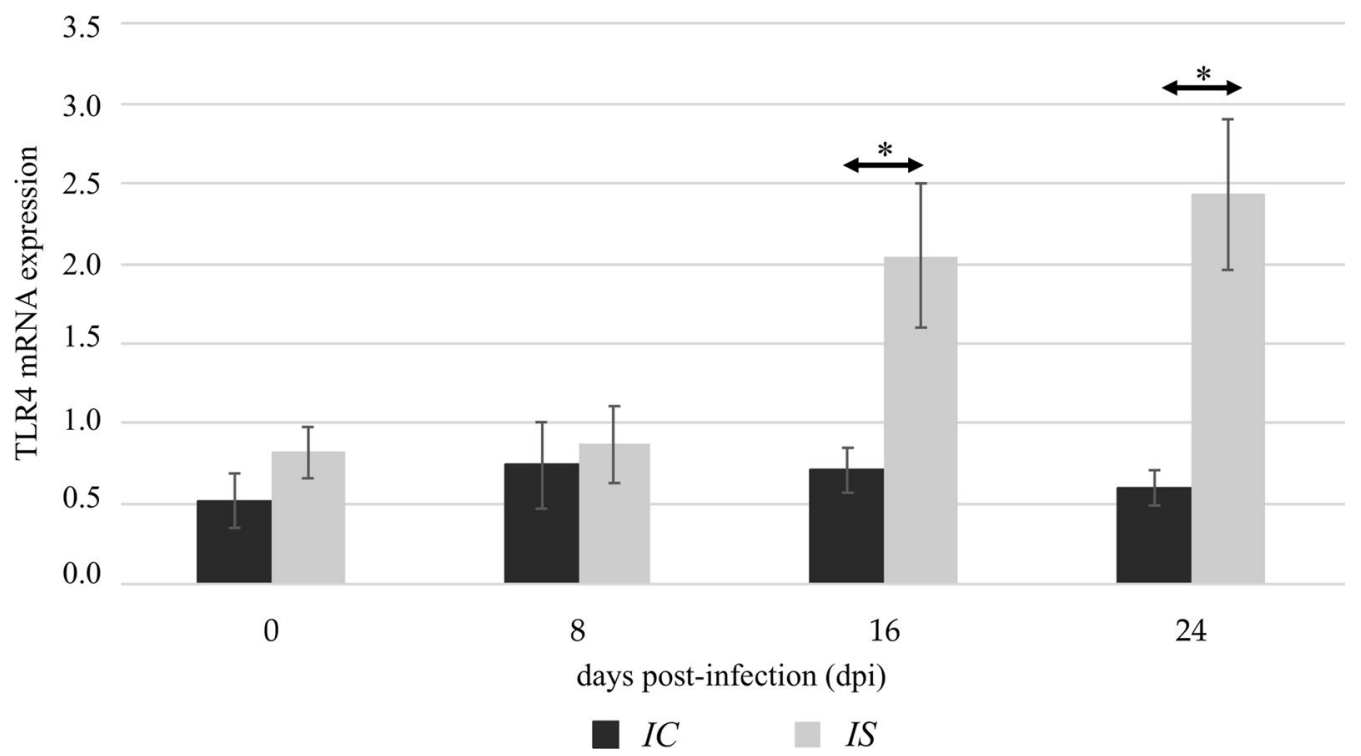

Fig. 2 The TLR4 mRNA expression in the kidneys of uninfected ( 0 dpi) and infected mice at 8, 16, and $24 \mathrm{dpi}$, in accordance with the immunological status of hosts (IC, immunocompetent mice; IS, immunosuppressed mice). The results are the means and standard deviations (SD) of six independent experiments; ${ }^{*} P<0.05$ using a Mann-Whitney U-test

The number of immunopositive cells and intensity of immunohistochemical reaction indicate a higher TLR4 expression in immunosuppressed uninfected mice than in immunocompetent uninfected mice (Fig. 6i, m).

\section{Discussion}

The immune response against parasitic infection is complex and involves many effectors and regulators components. Acanthamoeba spp. activate the classical TLR signaling pathway inducing NF-kB activation and increased secretion of inflammatory cytokines [24]. Induction of an inflammatory response by amoebae has been proposed as an important factor to determine the course of the parasite infection [8]. Nevertheless, little is known about the innate immune response induced by Acanthamoeba spp. in the kidneys and heart. To our knowledge, this is the first report showing TLRs expression in the kidneys and heart of hosts with disseminated acanthamoebiasis. Hence, further knowledge about the molecular and immunological mechanisms induced by amoebae are important aspects to understand the course of infections and tissue invasion.

The occurrence of Acanthamoeba spp. infection has been described in patients following kidney transplantation [25-28], although in just one instance the amoebae were re-isolated from the kidney of a patient with probable Acanthamoeba meningoencephalitis [29]. In our experimental model, despite the re-isolation of Acanthamoeba spp. from the mouse kidneys, the kidney profile performed in mouse serum indicated normal renal function [7]. In the study concerning histopathological changes in the kidneys of mice infected with Acanthamoeba spp. strain AM22, we observed elevated proliferation of cellular nuclei in the proximal/distal tubule epithelium and areas with less acidic cytoplasm (unpublished data). In the kidneys of mice infected with an environmental isolate of Acanthamoeba spp., Górnik \& Kuźna-Grygiel [30] observed haemorrhages, inflammatory foci, and even necrotic changes in the renal tubules and Bowman's capsules. Therefore, based on previous studies, the affinity of Acanthamoeba spp. to the kidneys can neither be confirmed nor excluded.

The factors involved in kidney damage and abnormal kidney function in parasitic diseases are still unknown. Studies on visceral leishmaniasis associated with renal abnormalities, suggest that Leishmania spp. antigens induce kidney inflammation by activating TLR2 and TLR4 receptors. The results indicate that kidney inflammatory processes and apoptosis involving TGF- $\beta$ have a significant role in the pathomechanism of kidney damage in Leishmania donovani infection [31]. However, based on studies on the role of Toll-like receptors in nephropathy induced by a Toxoplasma gondii infection, it was found that mainly TLR2 plays a role in kidney protection against T. gondii infection. Histopathological studies showed larger kidney damage in TLR2 deficient mice compared to TLR4 deficient mice [32]. In the present study, we found upregulation of both TLRs in immunocompromised Acanthamoeba spp.-infected mice, but statistically significant differences were only found 


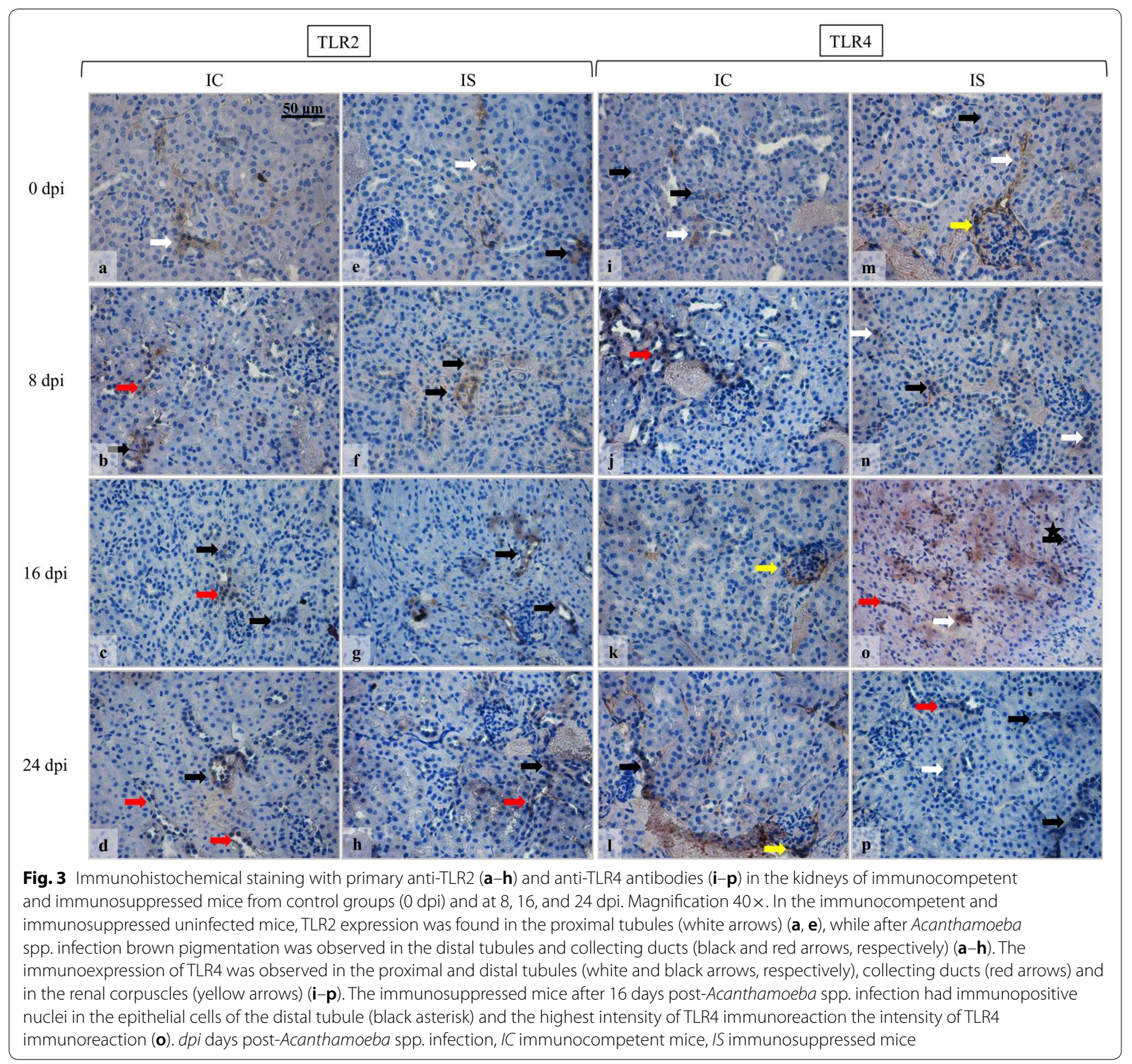

in TLR2 expression on the decline of infection. Higher TLRs expression at 16 and 24 dpi may be a result of used corticosteroid or the number of parasites that entered the kidneys. Mun et al. [33] observed that the effect of TLR2 on survival of T. gondii-infected mice depended on the infection dose. In our study, the mice were infected with 10-20 thousand amoebae, but Acanthamoeba spp. were re-isolated mostly from the kidneys of immunosuppressed mice at $16 \mathrm{dpi}$ and $24 \mathrm{dpi}$.

Leemans et al. [34] have shown that TLR2 plays a critical role in the initiation of acute renal inflammation and early tubular injury. Histological and morphological examination of Acanthamoeba spp.-infected kidneys revealed only elevated proliferation of nuclei in the renal tubules and a lighter color of the kidney parenchyma (unpublished data). It is possible that histopathological changes in the form of inflammatory infiltrates could have been visible in the kidneys of mice in a longer-lasting acanthamoebiasis; statistically significant changes in TLR2 expression were observed only at $24 \mathrm{dpi}$, the last day of the experiment. In future studies, the experiment should be extended to 30-40 days post-Acanthamoeba spp. infection and include determination of TGF- $\beta$ expression which induces transformation of kidney tubule cells to proliferating fibroblasts, causing fibrous changes in kidney parenchyma [35]. Such changes may 


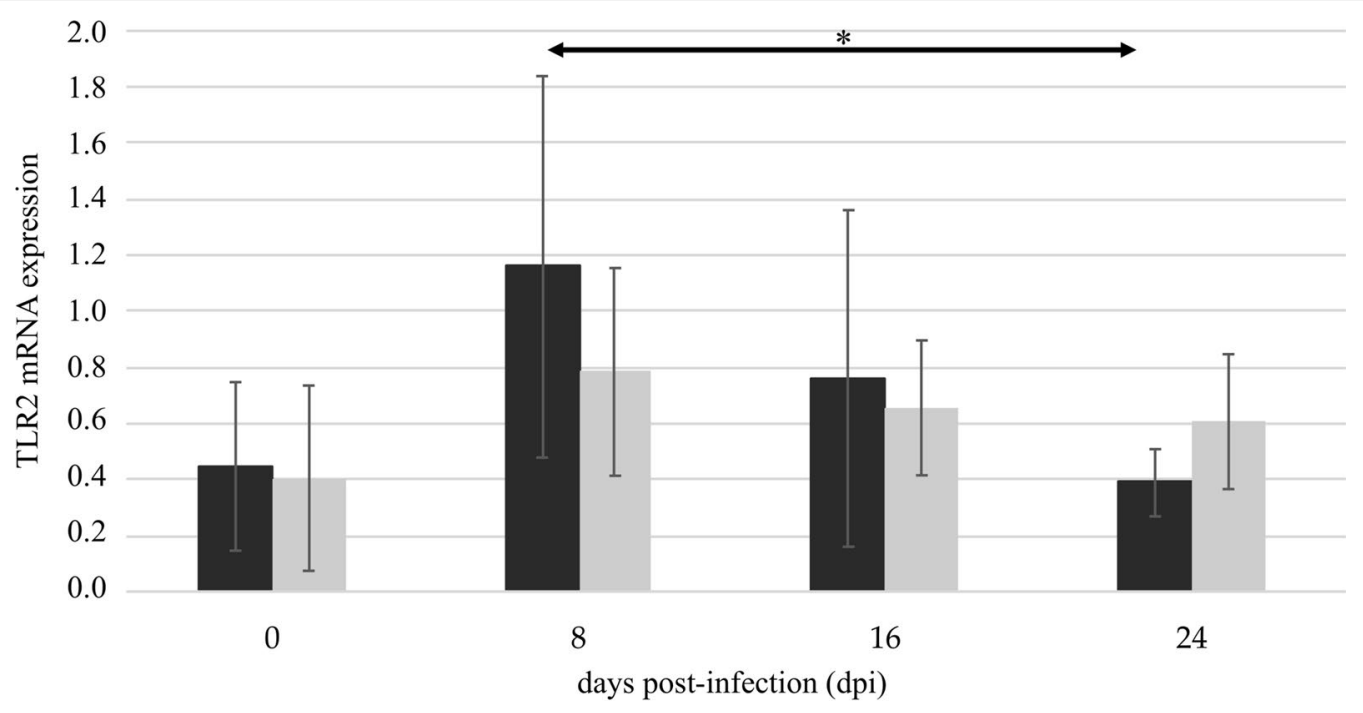

IC

IS

Fig. 4 The TLR2 mRNA expression in the heart of uninfected ( 0 dpi) and infected mice at 8, 16, and 24 dpi, in accordance with the immunological status of hosts (IC, immunocompetent mice; IS, immunosuppressed mice). The results are the means and standard deviations (SD) of six independent experiments; ${ }^{*} P<0.05$ using a Mann-Whitney U-test

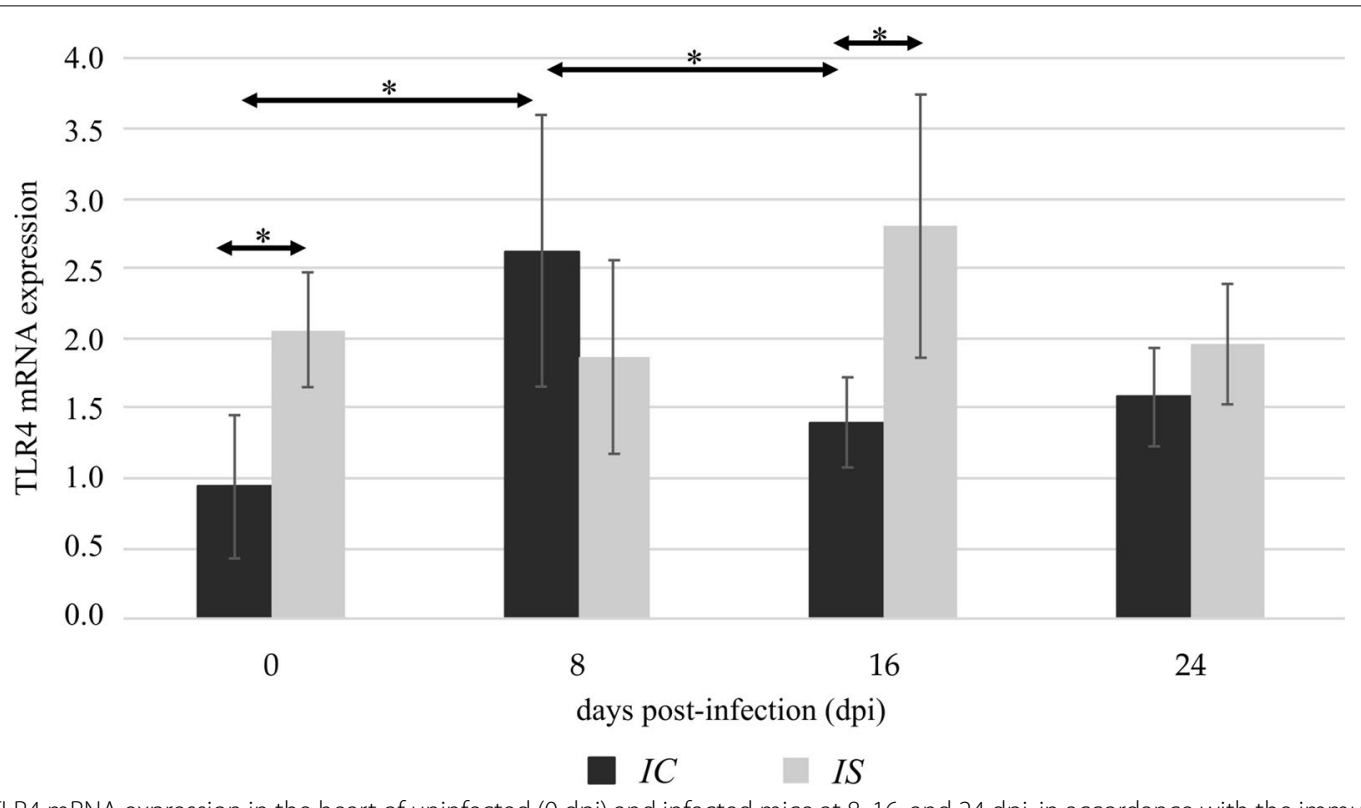

Fig. 5 The TLR4 mRNA expression in the heart of uninfected (0 dpi) and infected mice at 8, 16, and 24 dpi, in accordance with the immunological status of hosts (IC, immunocompetent mice; IS, immunosuppressed mice). The results are the means and standard deviations (SD) of six independent experiments; ${ }^{*} P<0.05$ using a Mann-Whitney $U$-test

be visible in histological preparations in the form of a lighter color of the kidney parenchyma.

The current literature presents three cases of cardiovascular patients with acanthamoebiasis [6, 36, 37], although no parasite forms have been found in cardiac muscle cells [37]. Also, in this study no trophozoites or cysts were found in myocardial histological preparations from Acanthamoeba spp.-infected mice, despite the fact that developmental forms of Acanthamoeba spp. were isolated from these samples (unpublished data). Therefore, 


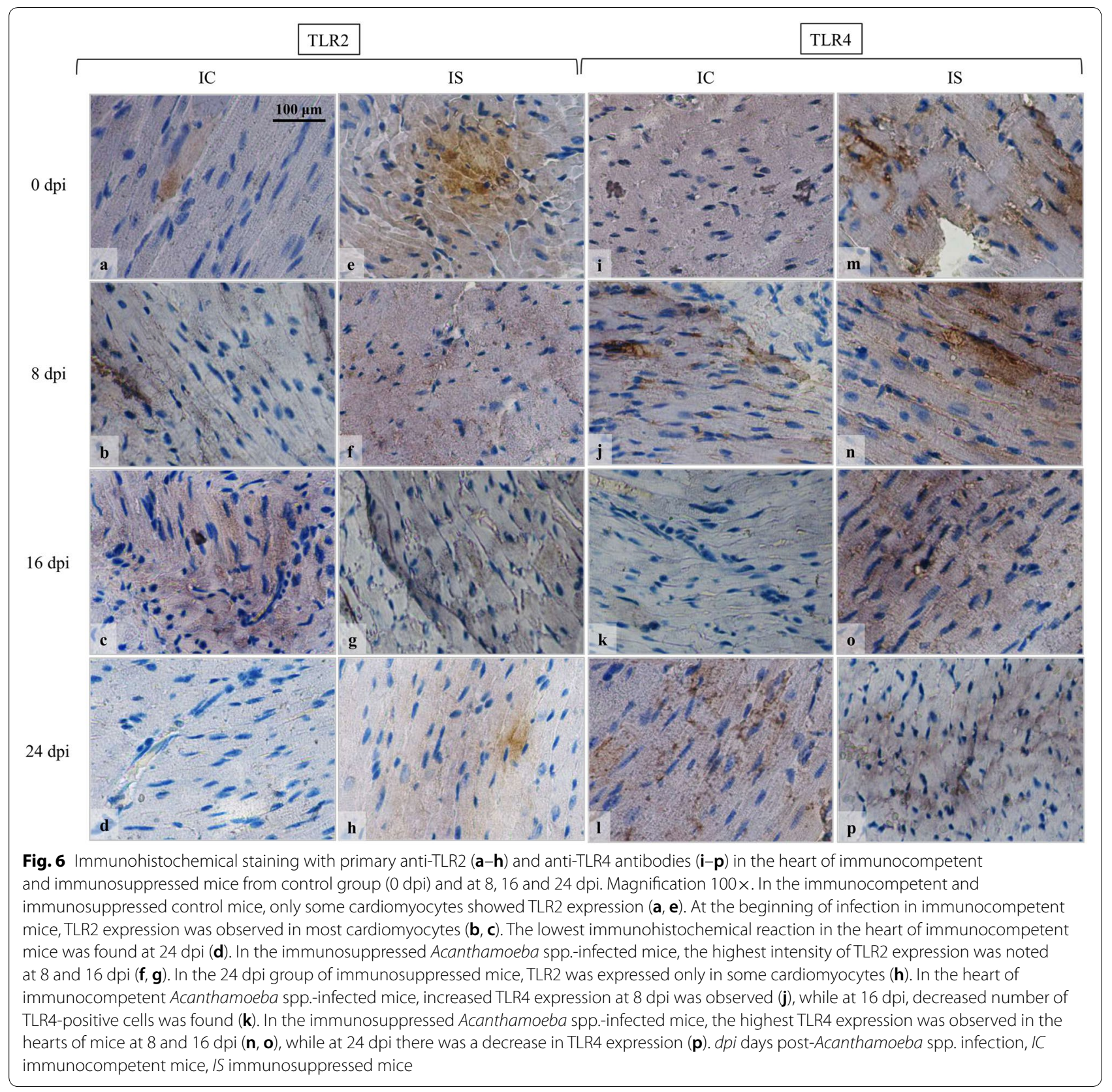

it is not clear whether the amoebae were re-isolated from cardiac fragments or from residual blood in the heart, as the amoebae spread throughout the host organism via the bloodstream.

The expression of Toll-like receptors has been reported in epithelium, endothelium and other cardiovascular cells $[38,39]$. The results of studies conducted so far suggest that short-term activation of TLR receptors has a protective effect on the cardiovascular system, while long-term or excessive activation of these receptors induces chronic inflammation [38-40]. Cardiomyocytes in response to inflammatory stimuli are capable of secreting pro- and anti-inflammatory cytokines capable of initiating and regulating the inflammatory response, as well as chemokines, which recruit and activate appropriate inflammatory cells [41]. The data on TLR expression in the cardiac muscle cells of infected hosts are scarce. Ponce et al. [42] observed increased tlr2 gene expression in the heart of BALB/c neonatal mice infected with Trypanosoma cruzi. The authors suggested that these parasites may activate the host's innate immune response via different Toll-like receptors to protect cardiomyocytes from parasites. In contrast, Pereira et al. [43] suggest that a high TLR2 expression in patients with chronic 
Chagas cardiomyopathy may induce an increase in IL-1 $\beta$, IL-12 and TNF- $\alpha$, thereby elevating cardiac inflammation and contributing to heart dysfunction. Oliveira et al. [44] observed that a deficiency of TLR4 leads mice to being more susceptible to T. cruzi infection, as evidenced by a higher parasitemia and earlier mortality. However, it is important to point out that $T$. cruzi has an affinity for the heart, which is not the main biotope of Acanthamoeba spp. In the present study, a significant upregulation of TLR2 expression was seen in the heart of immunocompetent mice at the beginning of infection. Additionally, we observed a significant increase in TLR4 expression in the heart of immunocompetent mice at the beginning of infection, followed by a statistically significant reduction at $16 \mathrm{dpi}$. This shows that TLR2 and TLR4 induced an immune response at 8 days post-Acanthamoeba spp. infection and thus protected cardiomyocytes from parasites, as confirmed by histological studies which did not show morphological changes (unpublished data).

Lack of statistically significant changes in TLRs expression in the heart of immunosuppressed mice may be involved with used corticosteroid. Mogensen et al. [45] concluded that dexamethasone, an immunosuppressive drug, inhibits TLR-receptor signaling in Neisseria meningitidis and Streptococcus pneumoniae invasion. Ma et al. [46] examining cardiomyopathy in mice, found that blocking TLR2 activity blunted cardiac dysfunction and inhibited cardiac fibrosis, whereas blocking TLR4 exacerbated cardiac dysfunction and fibrosis. Histological studies of Acanthamoeba spp.-infected mice treated with an immunosuppressive drug showed morphological changes in the form of hemorrhages and vacuolized cardiomyocytes with less acidic cytoplasm at $8 \mathrm{dpi}$ and $16 \mathrm{dpi}$. At 24 dpi, we observed no morphological changes in the cardiac muscle (unpublished data), which might have been caused by slightly increased TLR4 expression in mice treated with the immunosuppressive drug at $16 \mathrm{dpi}$.

\section{Conclusions}

The immunological mechanisms preventing renal and cardiomyocyte pathomechanisms in Acanthamoeba spp. infection remain unknown. The present study showed upregulation in TLRs in the kidneys and heart of hosts with disseminated acanthamoebiasis. Our results indicate that TLR2 is involved in response to Acanthamoeba spp. infection in the kidneys, whereas in the heart, both studied TLRs are involved in response to Acanthamoeba spp. infection. In future studies, it will be important to analyze the cytokine profile in the heart and kidneys of hosts with disseminated acanthamoebiasis to better understand the course of infection and tissue invasion.

\section{Abbreviations}

A: immunocompetent Acanthamoeba spp.-infected mice; AK: Acanthamoeba keratitis; AM 22: amoebic strain no. 22; AM: arithmetic mean; AS: immunosuppressed by methylprednisolone sodium succinate Acanthamoeba spp.infected mice; C: immunocompetent uninfected mice, immunocompetent control group; CS: immunosuppressed by methylprednisolone sodium succinate uninfected mice, immunosuppressed control group; DAMPs: damageassociated molecular patterns; dpi: days post-infection; GAE: granulomatous amebic encephalitis; HSP60: heat shock protein 60; HSP70: heat shock protein 70; IC: immunocompetent mice; IS: immunosuppressed mice; IL-1 $\beta$ : interleukin 1 3 ; IL-8: interleukin 8; IL-12: interleukin 12; IFN- $\gamma$ : interferon gamma; IRF: interferon regulatory factor; MPS: methylprednisolone sodium succinate; NF-KB: nuclear factor kappa-light-chain-enhancer of activated B cells; NN: nonnutrient agar; $P$ : level of significance; PAMPs: pathogen-associated molecular patterns; PBS: phosphate-buffered saline; SD: standard deviation; TLR2: toll-like receptor 2; TLR4: toll-like receptor 4; TGF- $\beta$ : transforming growth factor $\beta$; TNFa: tumor necrosis factor alpha.

\section{Acknowledgements}

Not applicable.

\section{Authors' contributions}

KK, DKB and NŁA conceived and designed research. KK, DKB, AWG, AKW and $\mathrm{N} Ł A$ performed the experiments. KK, AWG and AKW analyzed the data. KK and DKB contributed to writing the manuscript. DKB and NŁA provided scientific supervision of the study. All authors read and approved the final manuscript.

\section{Funding}

The Pomeranian Medical University in Szczecin provided financial support (WLBiML-430-01/S/14/2020).

\section{Availability of data and materials}

The datasets used and analysed during the present study are available from the corresponding author upon reasonable request.

\section{Ethics approval and consent to participate}

Consent from the Local Ethics Committee for Scientific Experiments on Animals in Szczecin (No. 29/2015 of 22 June 2015) and Poznań (No. 64/2016 of 9 September 2016) were obtained to conduct the experiment on laboratory animals. All animal experiments were performed in strict agreement with good animal practice with the recommendations in the Guide for Care and Use of Laboratory Animals.

\section{Consent of publication}

Not applicable.

\section{Competing interests}

The authors declare that they have no competing interests.

\section{Author details}

${ }^{1}$ Department of Biology and Medical Parasitology, Pomeranian Medical University in Szczecin, Powstancow Wielkopolskich 72, 70-111 Szczecin, Poland. ${ }^{2}$ Independent of Pharmaceutical Botany, Department of Biology and Medical Parasitology, Pomeranian Medical University in Szczecin, Powstancow Wielkopolskich 72, 70-111 Szczecin, Poland. ${ }^{3}$ Department of Biology and Medical Parasitology, Poznan University of Medical Sciences, Fredry 10, 61-701 Poznan, Poland. ${ }^{4}$ Department of Histology and Embryology, Pomeranian Medical University in Szczecin, Powstancow Wielkopolskich 72, 70-111 Szczecin, Poland.

Received: 26 June 2020 Accepted: 11 September 2020

Published online: 21 September 2020

\section{References}

1. Khan NA. Acanthamoeba: biology and increasing importance in human health. FEMS Microbiol Rev. 2006;20:564-95.

2. Nair KV, Gillon J, Ferguson A. Corticosteroid treatment increases parasite numbers in murine giardiasis. Gut. 1981;22:475-80. 
3. Kot K, Łanocha-Arendarczyk N, Kosik-Bogacka DI. Amoebas from the genera Acanthamoeba and their pathogenic properties. Ann Parasitol. 2018;64:299-308.

4. Visvesvara GS, Moura H, Schuster FL. Pathogenic and opportunistic free-living amoebae: Acanthamoeba spp., Balamuthia mandrillaris, Naegleria fowleri and Sappinia diploidea. FEMS Immunol Med Microbiol. 2007;56:1-26.

5. Łanocha-Arendarczyk N, Kosik-Bogacka D, Galant K, Zaorski W, Kot K, Łanocha A. Pathogenic free-living amoeba. Post Mikrobiol. 2017:56:106-12

6. Brondfield MN, Reid MJ, Rutishauser RL, Cope JR, Tang J, Ritter JM, et al. Disseminated Acanthamoeba infection in a heart transplant recipient treated successfully with a miltefosine-containing regimen: case report and review of the literature. Transpl Infect Dis. 2017. https://doi. org/10.1111/tid.12661.

7. Łanocha-Arendarczyk N, Baranowska-Bosiacka I, Kot K, Pilarczyk B, TomzaMarciniak A, Kabat-Koperska J, et al. Biochemical profile, liver and kidney selenium (Se) status during acanthamoebiasis in a mouse model. Folia Biol. 2018;66:33-40.

8. Łanocha-Arendarczyk N, Kolasa-Wołosiuk A, Wojciechowska-Koszko I, Kot K, Roszkowska P, Krasnodębska-Szponder B, et al. Changes in the immune system in experimental acanthamoebiasis in immunocompetent and immunosuppressed hosts. Parasit Vectors. 2018;11:517.

9. Łanocha-Arendarczyk N, Baranowska-Bosiacka I, Kot K, Gutowska I, Kolasa-Wołosiuk A, Chlubek D, et al. Expression and activity of COX-1 and COX-2 in Acanthamoeba sp.-infected lungs according to the host immunological status. Int J Mol Sci. 2018;19:E121.

10. Łanocha-Arendarczyk N, Baranowska-Bosiacka I, Gutowska I, KolasaWołosiuk A, Kot K, Łanocha A, et al. The activity of matrix metalloproteinases (MMP-2, MMP-9) and their tissue inhibitors (TIMP-1, TIMP-3) in the cerebral cortex and hippocampus in experimental acanthamoebiasis. Int J Mol Sci. 2018;19:E4128.

11. Kot K, Kosik-Bogacka D, Łanocha-Arendarczyk N, Wojtkowiak-Giera A, Kolasa-Wołosiuk A. Expression of Toll-like receptors (TLR2 and TLR4) in the eyes of mice with disseminated acanthamoebiasis. Biomed Res Int. 2019;2019:1401894

12. Termeer C, Benedix F, Sleeman J, Fieber C, Voith U, Ahrens T, et al. Oligosaccharides of hyaluronan activate dendritic cells via toll-like receptor 4 . J Exp Med. 2002;195:99-111.

13. Siddiqui R, Khan NA. Biology and pathogenesis of Acanthamoeba. Parasit Vectors. 2012;4:6.

14. Ohashi K, Burkart V, Flohé S, Kolb H. Cutting edge: heat shock protein 60 is a putative endogenous ligand of the toll-like receptor-4 complex. J Immunol. 2000;164:558-61.

15. Grygorowicz MA, Kozłowska E. Involvement of receptors recognizing pathogen-associated molecular patterns - TLRs in modulation of regulatory T cell CD4 +CD25 +FoxP3 + activity. Post Mikrobiol. 2011;50:141-54.

16. Cano A, Mattana A, Woods S, Henriquez FL, Alexander J, Roberts CW. Acanthamoeba activates macrophages predominantly through Toll-like receptor 4- and MyD88-dependent mechanisms to induce interleukin-12 (IL-12) and IL-6. Infect Immun. 2017;85:e01054-116.

17. Mattana A, Sanna M, Cano A, Delogu G, Erre G, Roberts CW, et al. Acanthamoeba castellanii genotype T4 stimulates the production of interleukin-10 as well as proinflammatory cytokines in THP-1 cells, human peripheral blood mononuclear cells, and human monocyte-derived macrophages. Infect Immun. 2016;84:2953-62.

18. Majewska M, Szczepanik M. The role of toll-like receptors (TLR) in innate and adaptive immune responses and their function in immune response regulation. Postepy Hig Med Dosw. 2006;60:52-63.

19. Alizadeh H, Tripathi T, Abdi M, Smith AD. Pathogenic strains of Acanthamoeba recognized by TLR4 and initiated inflammatory responses in the cornea. PLoS One. 2014;9:e92375.

20. Wojtkowiak-Giera A, Derda M, Kolasa-Wołosiuk A, Hadaś E, Kosik-Bogacka $D$, Solarczyk $P$, et al. Toll-like receptors in the brain of mice following infection with Acanthamoeba spp. Parasitol Res. 2016;115:4335-44.

21. Derda M, Wojtkowiak-Giera A, Kolasa-Wołosiuk A, Kosik-Bogacka D, Hadaś E, Jagodziński PP, et al. Acanthamoeba infection in lungs of mice expressed by toll-like receptors (TLR2 and TLR4). Exp Parasitol. 2016;165:30-4

22. Lanocha N, Kosik-Bogacka D, Maciejewska A, Sawczuk M, Wilk A, Kuzna-Grygiel W. The occurence Acanthamoeba (free-living amoeba) in environmental and respiratory tract samples in Poland. Acta Protozool. 2009;48:271-9.

23. Markowitz SM, Sobieski T, Martinez AJ, Duma RJ. Experimental Acanthamoeba infections in mice pretreated with methylprednisolone or tetracycline. Am J Pathol. 1978;92:733-44.

24. Neelam S, Niederkorn JY. Pathobiology and immunobiology of Acanthamoeba keratitis: insights from animal models. Yale J Biol Med. 2017;90:261-8

25. Steinberg JP, Galindo RL, Kraus ES, Ghanem KG. Disseminated acanthamebiasis in a renal transplant recipient with osteomyelitis and cutaneous lesions: case report and literature review. Clin Infect Dis. 2002;35:43-9.

26. Satlin MJ, Graham JK, Visvesvara GS, Mena H, Marks KM, Saal SD, et al. Fulminant and fatal encephalitis caused by Acanthamoeba in a kidney transplant recipient: case report and literature review. Transpl Infect Dis. 2013;15:619-26.

27. Salameh A, Bello N, Becker J, Zangeneh T. Fatal granulomatous amoebic encephalitis caused by Acanthamoeba in a patient with kidney transplant: a case report. Open Forum Infect Dis. 2015;2:ofv104.

28. Winsett F, Dietert J, Tschen J, Swaby M, Bangert CA. A rare case of cutaneous acanthamoebiasis in a renal transplant patient. Dermatol Online J. 2017;23:13030/qt88s2t7wp.

29. Ringsted J, Val Jager B, Suk DS, Visvesvara GS. Probable Acanthamoeba meningoencephalitis in a Korean child. Am J Clin Pathol. 1976;66:723-30.

30. Górnik K, Kuźna-Grygiel W. Histological studies of selected organs of mice experimentally infected with Acanthamoeba spp. Folia Morphol. 2005;64:161-7.

31. Kumar V, Tiwari N, Gedda MR, Haque R, Singh RK. Leishmania donovani infection activates Toll-like receptor 2, 4 expressions and transforming growth factor-beta mediated apoptosis in renal tissues. Braz J Infect Dis. 2017;21:545-9.

32. Kudo M, Aosai F, Mun HS, Norose $K$, Akira S, Iwakura Y, et al. The role of IFN-gamma and Toll-like receptors in nephropathy induced by Toxoplasma gondii infection. Microbiol Immunol. 2004;48:617-28.

33. Mun HS, Aosai F, Norose K, Chen M, Piao LX, Takeuchi O, et al. TLR2 as an essential molecule for protective immunity against Toxoplasma gondii infection. Int Immunol. 2003;15:1081-7.

34. Leemans JC, Butter LM, Pulskens WP, Teske GJ, Claessen N, van der Poll T, et al. The role of Toll-like receptor 2 in inflammation and fibrosis during progressive renal injury. PLoS One. 2009;4:e5704.

35. Robertson H, Ali S, McDonnell BJ, Burt AD, Kirby JA. Chronic renal allograft dysfunction: the role of T cell-mediated tubular epithelial to mesenchymal cell transition. J Am Soc Nephrol. 2004;15:390-7.

36. Barete S, Combes A, de Jonckheere JF, Datry A, Varnous S, Martinez V, et al. Fatal disseminated Acanthamoeba lenticulata infection in a heart transplant patient. Emerg Infect Dis. 2007;13:736-8.

37. Tan SK, Gajurel K, Tung C, Albers G, Deresinski S, Montoya JG, et al. Fatal Acanthamoeba encephalitis in a patient with a total artificial heart (syncardia) device. Open Forum Infect Dis. 2014;1:ofu057.

38. Frantz S, Ertl G, Bauersachs J. Mechanisms of disease: toll-like receptors in cardiovascular disease. Nat Clin Pract Cardiovasc Med. 2007;4:444-54.

39. Vallejo JG. Role of toll-like receptors in cardiovascular diseases. Clin Sci. 2011;121:1-10.

40. de Kleijn D, Pasterkamp G. Toll-like receptors in cardiovascular diseases. Cardiovasc Res. 2003;60:58-67.

41. Boyd JH, Mathur S, Wang Y, Bateman RM, Walley KR. Toll-like receptor stimulation in cardiomyoctes decreases contractility and initiates an NF-kappa B dependent inflammatory response. Cardiovasc Res. 2006;72:384-93.

42. Ponce NE, Cano RC, Carrera-Silva EA, Lima AP, Gea S, Aoki MP. Toll-like receptor-2 and interleukin-6 mediate cardiomyocyte protection 141 from apoptosis during Trypanosoma cruzi murine infection. Med Microbiol Immunol. 2012:201:145-55.

43. Pereira NS, Queiroga TBD, Nunes DF, Andrade CM, Nascimento MSL, DoValle-Matta MA, et al. Innate immune receptors over expression correlate with chronic chagasic cardiomyopathy and digestive damage in patients. PLoS Negl Trop Dis. 2018;12:e0006589.

44. Oliveira AC, Peixoto JR, de Arruda LB, Campos MA, Gazzinelli RT, Golenbock DT, et al. Expression of functional TLR4 confers proinflammatory responsiveness to Trypanosoma cruzi glycoinositolphospholipids and higher resistance to infection with T. cruzi. J Immunol. 2004;173:5688-96. 
45. Mogensen TH, Berg RS, Paludan SR, Østergaard L. Mechanisms of dexamethasone-mediated inhibition of Toll-like receptor signaling induced by Neisseria meningitidis and Streptococcus pneumoniae. Infect Immun. 2008;76:189-97.

46. Ma Y, Zhang X, Bao H, Mi S, Cai W, Yan H, et al. Toll-like receptor (TLR) 2 and TLR4 differentially regulate doxorubicin induced cardiomyopathy in mice. PLoS One. 2012;7:e40763.

\section{Publisher's Note}

Springer Nature remains neutral with regard to jurisdictional claims in published maps and institutional affiliations. 